Preface

\title{
Hearing Preservation and Auditory Rehabilitation in Skull Base Surgery
}

\author{
Matthew L. Carlson ${ }^{1,2}$ Daniel Jethanamest ${ }^{3}$ \\ ${ }^{1}$ Department of Otolaryngology-Head and Neck Surgery, Mayo \\ Clinic, Rochester, Minnesota, United States \\ ${ }^{2}$ Department of Neurosurgery, Mayo Clinic, Rochester, Minnesota, \\ United States \\ 3 Department of Otolaryngology-Head and Neck Surgery, NYU \\ Langone Health, New York, New York, United States
}

J Neurol Surg B 2019;80:109-110.

It is with great pleasure we present to you this special issue in Journal of Neurological Surgery Part B: Skull Base, entitled "Hearing Preservation and Auditory Rehabilitation in Skull Base Surgery." The management of benign lateral skull base tumors has evolved considerably over the last several decades. Driven by advances in microsurgical technique and low-dose highly conformal radiation, combined with an enhanced understanding of the natural history of tumor growth, the modern management of lateral skull base disease currently prioritizes function preservation over radical tumor removal. Under this paradigm, cranial nerve preservation is achieved in the majority of patients. Notwithstanding considerable advances in the field, durable preservation of functional hearing in patients with vestibular schwannoma and other lateral skull base tumors remains elusive.

This special issue focuses on hearing-related topics relevant to vestibular schwannoma, intralabyrinthine schwannoma, jugular parganglioma, and sequelae of temporal bone

radiation and systemic chemotherapy. Leaders and innovators in the field provide focused reviews on audiologic testing and hearing outcome reporting, tinnitus management, surgical, and nonsurgical techniques for rehabilitation of unilateral conductive hearing loss, single-sided deafness, bilateral profound hearing loss, and hearing preservation outcomes according to disease and management. We wish to sincerely thank all of our expert

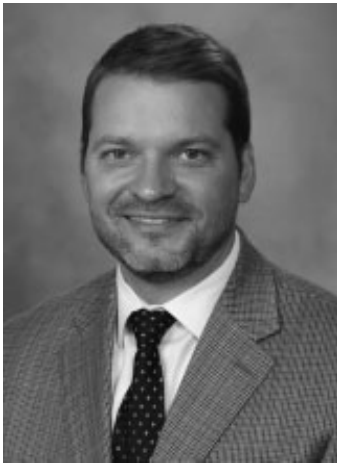

Matthew L. Carlson, MD

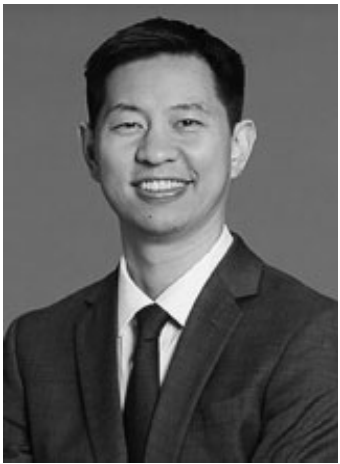

Daniel Jethanamest, MD contributors for their outstanding submissions and hard work which was essential to complete this task. We also wish to thank Journal Editors Dennis Kraus M.D. and Michael Link M.D. for the opportunity to be guest editors for this stimulating and challenging topic.

Address for correspondence Matthew L. Carlson, MD, Department of OtolaryngologyHead and Neck Surgery, Mayo Clinic, Rochester, Minnesota, United States (e-mail: Carlson.Matthew@mayo.edu).
(C) 2019 Georg Thieme Verlag KC Stuttgart · New York
DOI https://doi.org/ $10.1055 / \mathrm{s}-0039-1683881$. ISSN 2193-6331. 